\title{
Features of performance of concrete and reinforced concrete structures of industrial facilities operated under impact of increased and high temperatures
}

\author{
Stanislav Fomin ${ }^{1 *}$, Iryna Plakhotnikova ${ }^{1}$, and Serhii Butenko ${ }^{1}$ \\ ${ }^{1}$ Department of reinforced concrete and stone structures, Kharkiv National University of Civil \\ Engineering and Architecture, 61002, Kharkiv, 40, Sumskaya Str., Ukraine
}

\begin{abstract}
Currently, the Normative base of Ukraine has moved to European standards. The process of harmonization with the Eurocodes was completed, new national normative documents of DBN and DSTU were developed and put into effect. At the same time it was turned out that the big section "Concrete and reinforced concrete structures intended for work in conditions of increased and high temperatures" is absent in the Eurocodes. By order of the Ministry of Regional Development, Construction and Housing and Communal Services of Ukraine, the development of a normative act is provided for the replacement of SNiP 2.03.04-84 with DBN with a change in status. Heat-resistant concretes in the elements of thermal aggregates are used in the iron and steel industry (blast furnaces, air heaters, coke batteries, etc.), non-ferrous metallurgy (graphite furnaces, fluidized bed furnaces, etc.), oil refining and petrochemical industry (tubular furnaces, vertical sectional furnaces, etc.), in the industry of building materials, and in various sectors of industry. These norms and rules apply to the design of concrete and reinforced concrete structures designed to operate under conditions of systematic exposure to increased $\left(50\right.$ to $200{ }^{\circ} \mathrm{C}$ inclusive) and high (above $200{ }^{\circ} \mathrm{C}$ ) process temperatures.
\end{abstract}

\section{Analysis of recent studies}

When developing a normative act for the replacement of SNiP 2.03.04-84 with DBN with a change in status, it is advisable to use the Book of Rules of SP 27.13330.2011 "Concrete and reinforced concrete structures intended for operation under increased and high temperatures."

This set of rules is built on the basis of numerous theoretical and experimental studies of K.D. Nekrasov [1], V.I. Belskiy [2], A.B. Toturbiev [3], G.F. Gitman, T.N. Malkina, A.F. Milovanov [4, 5], A.E. Desov [6], R. Von der Heyden, P. Fritsch, G. Krishtanits, R. Rumpl [7], and others.

${ }^{*}$ Corresponding author: $\underline{\text { sfomin } @ \text { ukr.net }}$ 
The rules were developed by NIIZhB named after A.A. Gvozdev - the institute of JSC "Research and Development Center "Stroitelstvo": the head - Grand PhD in Engineering Sciences, prof. A.F. Milovanov Executors: Grand PhDs in Engineering Sciences, prof. A.P. Krichevskiy, S.L. Fomin; PhDs in Engineering Sciences V. N. Goryachev, N.P. Zhdanova, I.M. Zaslavskiy, V.N. Milonov, V.G. Petrov-Denisov, V. N. Samoylenko, V.V. Solomonov, I.S. Kuznetsova, engineers E.M. Bolnykh, V.A. Tarasov, with the participation of LLC "UralNIIstrom" (PhD in Engineering Sciences R.Ya. Akhtyamov) [8].

The papers on the coke-chemical industry for the foundations of coke batteries are also evidence of the need to create DBN. In papers $[9,10]$, we obtained temperature fields in reinforced concrete structures, found new features of the stressed-deformed state of reinforced concrete monolithic structures of coke furnace batteries, and obtained results of experimental studies that revealed a number of new features of the work of reinforced concrete structures and allowed to improve the nature of reinforcement, to prove the feasibility of constructive solutions of foundations of heat-resistant concrete without lining of breaching.

\section{The results of the theoretical studies performed}

The method of calculation of reinforced concrete elements operating under conditions of the technological environment includes the evaluation of heat and moisture transfer, free temperature and humidity deformations; creep, shrinkage, full diagrams of " $\sigma-\varepsilon$ " concrete and reinforcement, stress and strain state of reinforced concrete elements.

The process of heat spreading in concrete can be approximately described by a scheme, according to which concrete is divided by an evaporation surface with a temperature of $100{ }^{\circ} \mathrm{C}$ into two zones: $1^{\text {st }}$ - dry and $2^{\text {nd }}$ - wet, the sizes of which continuously change with increase of temperature. In this case, the thermophysical characteristics of each of the zones are different functions of temperature.

The determination of the temperature field of such a two-zone environment must be carried out taking into account the variable position of the interface of the zones, as well as the continuous absorption of latent heat of vaporization in the zone of separation (in analogy with the Stefan problem):

for dry zone

$$
\rho C(t) \frac{\partial t_{1}}{\partial \tau}+\rho \frac{\partial C(t)}{\partial \tau} t_{1}=\nabla\left[\lambda(t) \nabla t_{1}\right]
$$

for wet zone

$$
\rho C(t) \frac{\partial t_{2}}{\partial \tau}+\rho \frac{\partial C(t)}{\partial \tau} t_{2}=\nabla\left[\lambda(t) \nabla t_{2}\right]
$$

At the interface between zones, the following condition must be met:

$$
-\lambda_{1} \nabla t_{1}(\xi, \tau)+\lambda_{2} \nabla t_{2}(\xi, \tau)=r W \rho \frac{\partial \xi}{\partial \tau}
$$

The impact of the technological environment is also manifested in the danger of brittle fracture of concrete [11]. The explosive brittle failure should be excluded, otherwise its impact on operational requirements should be taken into account (Fig. 1).

The explosive brittle failure is unlikely if the concrete moisture content is less than $k \%$. The recommended value of $k=3 \%$. If the moisture value is more than $k \%$, more accurate 
assessment of the moisture content, type of aggregate, water permeability of concrete and heating conditions should be carried out.

During high-temperature intensive heating, a gradient of the total pressure of moist air arises inside the wet concrete, resulting in an additional transfer of moisture and heat due to the presence of hydrodynamic (filtration) movement of vapour and liquid, while the diffusion transfer is negligibly small.

The mathematical model can be represented in the form of a system of nonlinear differential equations describing the development of the temperature and pressure field with internal sources and drains:

$$
\begin{aligned}
& C_{t} \rho \frac{\partial t}{\partial \tau}=\nabla\left[\lambda_{t} \nabla t\right]+r_{t} \rho B_{t} \frac{\partial t}{\partial \tau} \\
& C_{p} \rho \frac{\partial p}{\partial \tau}=\nabla\left[\lambda_{p} \nabla p\right]-\rho B_{t} \frac{\partial t}{\partial \tau}
\end{aligned}
$$

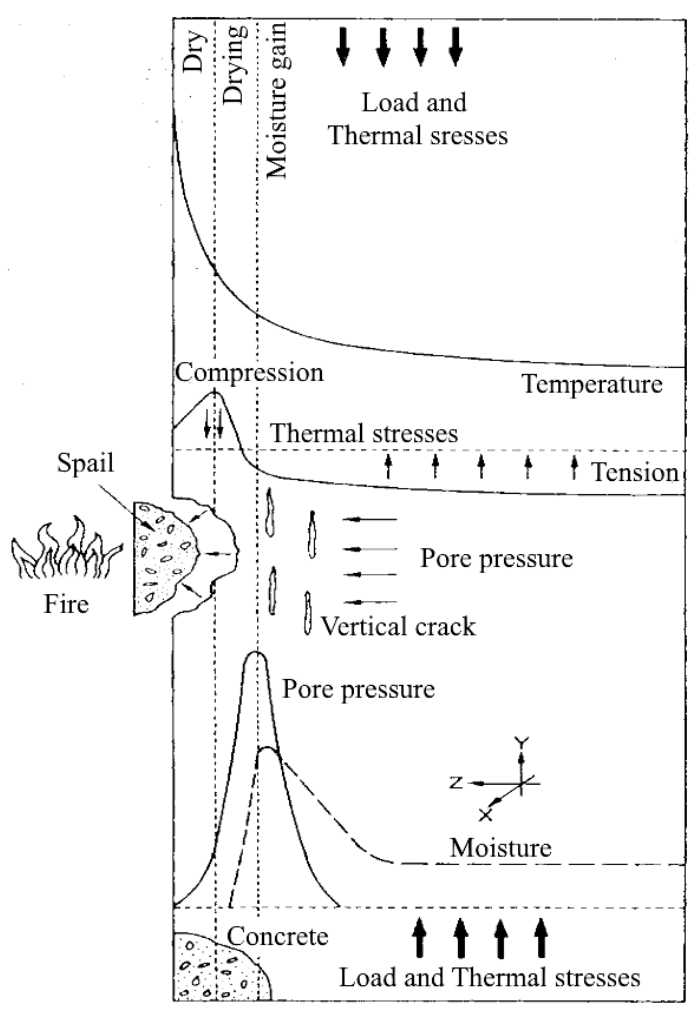

Fig. 1.The explosive peeling of concrete under the influence of pore pressures and thermal stresses [11].

$$
\frac{\partial W}{\partial \tau}=B_{t} \frac{\partial t}{\partial \tau}
$$

A modification of the method of V.M. Roitman [12-14] was proposed for determination of the phase transition intensity, the following formula was experimentally obtained: 


$$
\begin{aligned}
& B_{t}=\frac{\partial W}{\partial t}=-2698 \cdot 10^{-4}+4.72 \cdot 10^{-6} t-1.086075 \cdot 10^{-6} t^{2}+ \\
& +7.70504 \cdot 10^{-9} t^{3}-1.722365 \cdot 10^{-11} t^{4}
\end{aligned}
$$

The introduction of the characteristic of the intensity of phase transformations makes it possible to combine terms in the equations (4) - (6) with time derivatives, introducing the concept of equivalent heat capacity and the vapour capacity by writing them as follows:

$$
\begin{aligned}
C_{r e d, t}(t) \rho \frac{\partial t}{\partial \tau} & =\nabla\left[\lambda_{t} \nabla t\right] \\
C_{r e d, p}(t) \rho \frac{\partial p}{\partial \tau} & =\nabla\left[\lambda_{p} \nabla p\right] \\
C_{r e d, t} & =C_{t}-r_{t} B_{t} \\
C_{r e d, p} & =C_{p}-B_{t}
\end{aligned}
$$

With this approach, the system of equations (4) ... (6) breaks up into separate differential equations, each of which shall be solved successively: first, the heat conductivity equation (8), then, using the rate of change of temperature in the elementary volume and the intensity of phase transitions known from the heat conductivity equations, equation of the field of excess pressures (9) and, finally, the equation of moisture conductivity (6).

The coefficients of thermal conductivity and specific heat as a function of temperature and humidity are proposed to be determined by the formulas:

$$
\begin{aligned}
& \lambda=1.37\left(1+0.175 W-0.016 W^{2}\right)(1-0.000277 t) \\
& C=0.83\left(1+0.077 W-0.0007 W^{2}\right)(1+0.001 t)
\end{aligned}
$$

\section{A numerical method for solving a system of non-linear differential equations of heat-moisture conductivity using a conservative finite-difference scheme}

A numerical solution of equations in partial derivatives is considered in the papers of R.D. Rikhtmayer [15], R.D. Richtmyer and K. Morton [16], A.R. Mitchell [17], L.A. Kozdoba [18].

The building of a difference scheme can be regarded as a replacement for a continuous environment described by differential equations with some discrete analogue of it. At finite steps of the grid, the discrete model can differ markedly from the continuous medium. This difference generates parasitic effects of difference origin, which reduce the value of the difference solution. Therefore, it is important to build such schemes that retain their qualities on the grids. The appearance of oscillations of the increasing amplitude caused by superposition of perturbations (perturbations can be generated either by machine rounding errors or transverse motions in a real two-dimensional problem) on the solution due to a large time step is called dynamic instability, which can be eliminated by decreasing the time step, making it smaller than some "critical step in time." The appearance of a monotonically increasing error is called a static instability, which can be eliminated only by a transition to some other finite-difference scheme. 
The differential equations of mathematical physics - is a mathematical expression of the basic laws of conservation (mass, momentum, and energy). Therefore, it is reasonable to build a difference scheme so that analogues of these laws are also fulfilled in it. The schemes of this type are called conservative.

A conservative difference scheme should reflect the basic properties of a continuous medium, i.e. the scheme should provide, first of all, the fulfilment of difference analogues of the basic conservation laws [19-20].

The conservative form of the equations of motion of an inviscid compressible fluid was derived by R. Kurant and K.O. Friedrichs. Its practical application was first realized by Lax, who transformed the ordinary equations of hydrodynamics, in which the dependent variables were velocity, density, and temperature, into a system of equations, in which the quantities of motion, density and the specific internal energy of breaking serve as dependent variables. This system of equations reflects the essence of physical conservation laws and allows preserving integral flow characteristics in a finite-difference scheme. If the traditional differential equations are transformed so that the conservative quantities become the sought variables, then the use of conservative finite-difference schemes ensures the conservation of mass, momentum, and energy.

The experience shows that conservative schemes give more accurate results. N.E. Sairus and R. E. Falton found that for elliptic equations, a conservative scheme yields more accurate results than a non-conservative one. A finite-difference method is a conservative method, if it ensures the fulfilment of certain integral conservation laws that are valid for the original differential equations.

A.N. Tikhonov and A.A Samarskiy proposed an integro-interpolation method for building conservative difference schemes, and an example was built, where a nonconservative difference scheme that provides the second order of accuracy in the class of sufficiently smooth coefficients diverges in the class of discontinuous coefficients.

Based on the provisions of the theory of moisture conductivity by V.N. Bogoslovskiy [21-23], a modified system of differential equations of heat and moisture transfer in concrete was proposed:

$$
\begin{aligned}
& \rho \eta(\theta, t) \frac{\partial \theta}{\partial \tau}+\rho \frac{\partial[\eta(\theta, t)]}{\partial \tau} \theta=\nabla[\kappa(\theta, t) \nabla \theta] \\
& \rho C(\theta, t) \frac{\partial t}{\partial \tau}+\rho \frac{\partial C(\theta, t)}{\partial \tau} t=\nabla[\lambda(\theta, t) \nabla t]
\end{aligned}
$$

Equations (14) - (15) with boundary conditions compiled from data on the change in temperature and the moisture potential of the environment completely determine the problem of calculation of temperature and humidity fields in cross-sections of reinforced concrete structures.

We considered the building of a conservative difference scheme for equations (14) (15), which is represented in the form

$$
\begin{gathered}
\frac{\partial t}{\partial \tau}=a \nabla^{2} t \\
\frac{\partial W}{\partial \tau}=\frac{1}{\rho} \nabla[\kappa(t, W) \nabla \theta]
\end{gathered}
$$


In equation (4), the coefficient of moisture conductivity $k$ is considered as a given moisture function $W$ and temperature $t$.

The computer programs have been developed for the calculation of the models presented.

\section{Conclusions}

The study of the concrete and reinforced concrete structures of industrial constructions operating under high and high temperatures was performed to create a new national normative act based on the latest achievements of science, engineering and technology, advanced domestic and foreign experience of designing and building and using the accumulated theoretical and experimental base of the SNiP 2.03.04-84.

This paper considers the impact of the technological environment on the physical and technical characteristics of building structures, including the emergence of the danger of brittle fracture of concrete.

During intensive high-temperature heating, a gradient of the total pressure of moist air arises inside the wet concrete, resulting in an additional transfer of moisture and heat due to the presence of hydrodynamic (filtration) movement of vapour and liquid, while the diffusion transfer is negligibly small.

The mathematical model was built in the form of a system of nonlinear differential equations describing the development of the temperature and pressure field with internal sources and drains. A conservative difference scheme was used, which should reflect the basic properties of a continuous medium, i.e. the scheme should provide, first of all, the fulfilment of difference analogues of the basic conservation laws. Based on the provisions of the theory of moisture conductivity by V.N. Bogoslovskiy, a modified system of differential equations of heat and moisture transfer in concrete was proposed. We considered the building of a conservative difference scheme for equations of heat and mass transfer, and the computer programs was developed.

\section{References}

1. K.D. Nekrasov, Refractory concretes, their properties and applications, Stroyizdat, Moscow (1949)

2. V.I. Belskiy and others. Construction of industrial furnaces and pipes of heat-resistant concrete, Stroyizdat, Moscow (1962)

3. A.B. Toturbiev, Concrete and reinforced concrete, 4, 10 (2011)

4. G.F. Gitman, T.N. Malkina, A.F. Milovanov, Heat-resistant concrete and reinforced concrete and areas of their effective use, VIIGH, Volgograd (1969)

5. A.F. Milovanov, Heat resisting reinforced concrete, Stroyizdat, Moscow (1963)

6. A.E. Desov, K.D. Nekrasov, A.F. Milovanov, Concretes for nuclear reactors, Cube and prismatic strength of concrete at high temperatures, Detroit, II (1972)

7. R. Von Der Heide, P. Fritsch, R. Krischyanits, P. Rampl, Cement and its application, 1, 200 (2012)

8. SP 27.13330.2011. Code of rules Concrete and reinforced concrete structures intended for work in conditions of increased and high temperatures. Updated version of SNiP 2.03.04-84, Moscow (2011)

9. B.A. Krylov, Studies in the field of heat-resistant concrete, Stroyizdat, Moscow (1981)

10. A.M. Kravchenko, L.N. Borisova, Handbook of the coke-chemist, Kharkov, (2016)

11. G.A. Khoury, C.E. Majjrana, Effect heat on concrete, Imperial College, London (2006) 
12. V.M. Roitman, A.G. Apostolov, T.S. Simonova, Fire resistance of building structures, VNIIPO, Moscow, 1, 220 (1973)

13. V.M. Roitman, A.T. Apostolov, T.S. Simonova. Fire resistance of building structures, VNIIPO, Moscow, 1, 130 (1973)

14. V.M. Roitman, T.N. Zyrina, Fire resistance of building structures, VNIIPO, Moscow, 58 (1974)

15. R. Richthmaier, Difference methods for solving boundary value problems, Mir, Moscow (1970)

16. R. Richthmyer, K. Morton, Difference methods for solving boundary value problems, Mir, Moscow (1972)

17. A.R. Mitchell, Computational methods in partial differential equations, J. Wiley and Sons Ltd, (1969)

18. L.A. Kozdoba, Solutions of nonlinear heat conduction problems, Naukova Dumka, Kiev (1976)

19. A.N. Tikhonov, A.A. Samarskiy, On the convergence of difference schemes in the class of discontinuous coefficients, DAN USSR, 3 (1959)

20. A.N. Tikhonov, A.A. Samarskiy, ZhVM MF, 1, 562 (1961)

21. V.N. Bogoslovskiy, Construction Thermophysics, High School, Moscow (1970)

22. V.N. Bogoslovsky, IFZh, 8, 31 (1965)

23. V.N. Bogoslovsky, Building Thermophysics, High School, Moscow (1982) 